INPLASY

PROTOCOL

To cite: You et al. Effectiveness and safety of acupuncture for chronic obstructive pulmonary disease: A protocol for systematic review and metaanalysis. Inplasy protocol

2021120081. doi:

10.37766/inplasy2021.12.0081

Received: 18 December 2021

Published: 18 December 2021

Corresponding author:

Tieming Ma

Inzymtm@163.com

Author Affiliation:

Liaoning University of

Traditional Chinese Medicine.

Support: XLYC1902004.

Review Stage at time of this submission: Preliminary searches.

Conflicts of interest:

None declared.

\section{Effectiveness and safety of acupuncture for chronic obstructive pulmonary disease: A protocol for systematic review and meta-analysis}

You, T1; Zhang, H2; Li, J3; Ma, T4.

Review question / Objective: This systematic review and meta-analysis will comprehensively summarize the latest published clinical evidence of acupuncture alone or combined with other interventions in the treatment of COPD in remission stage.

Condition being studied: Chronic Obstructive Pulmonary Disease (COPD) is the most common respiratory disease in clinic. Traditional Chinese Medicine(TCM) lung rehabilitation has gradually been valued in the field of prevention and treatment of COPD. Acupuncture, as an important part of TCM lung rehabilitation, is carried out in clinical extensively and application. It has the advantage of improving the symptoms and quality of life of COPD cases, but the evidence-based proof is limited.

INPLASY registration number: This protocol was registered with the International Platform of Registered Systematic Review and Meta-Analysis Protocols (INPLASY) on 18 December 2021 and was last updated on 18 December 2021 (registration number INPLASY2021120081).

\section{INTRODUCTION}

Review question / Objective: This systematic review and meta-analysis will comprehensively summarize the latest published clinical evidence of acupuncture alone or combined with other interventions in the treatment of COPD in remission stage.

Condition being studied: Chronic Obstructive Pulmonary Disease (COPD) is the most common respiratory disease in clinic. Traditional Chinese Medicine(TCM) 
lung rehabilitation has gradually been valued in the field of prevention and treatment of COPD. Acupuncture, as an important part of TCM lung rehabilitation, is carried out in clinical extensively and application. It has the advantage of improving the symptoms and quality of life of COPD cases, but the evidence-based proof is limited.

\section{METHODS}

Participant or population: Patients should be diagnosed as stable COPD by guidelines or expert consensus with clear diagnostic criteria.

Intervention: The intervention measures in control group can include sham acupuncture, routine treatment of western medicine, Chinese medicine, acupoint application, moxibustion and so on. The intervention measures in treatment group should be combined with acupuncture or acupuncture combined with other intervention measures on the basis of control group. Acupuncture mainly includes body acupuncture, scalp acupuncture, eye acupuncture, ear acupuncture, etc. There will be no restrictions on acupoint selection, time and course of treatment.

Comparator: The intervention measures in control group can include sham acupuncture, routine treatment of western medicine, Chinese medicine, acupoint application, moxibustion and so on.

Study designs to be included: Randomized controlled trials.

Eligibility criteria: We will not emphasize whether blind method is adopted in the execution of clinical research. Observational studies, animal experiments, literature review, case reports, repeatedly published articles, experience summary, protocol, etc. will be excluded.

Information sources: We will retrieve the following 8 databases: PubMed, EMBASE, Cochrane Central Register of Controlled Trials, Web of Science, China National Knowledge Infrastructure, WangFang
Database, Chinese Science and Technology Periodical Database, SinoMed.The retrieval time is from the establishment of each database to December 2021.

Main outcome(s): Clinical effective rate; TCM symptom score; quality of life (COPD assessment test, St. George respiratory questionnaire, chronic respiratory questionnaire, etc); dyspnea (borg scale, visual analog dyspnea scale, modified British medical research council respiratory diffificulty questionnaire, etc); exercise capacity (6-minute walking test, shuttle walking test, etc); lung function (FEV1, FVC, FEV1/FVC, TLC, RV, etc); frequency of acute exacerbation; adverse effects.

Quality assessment / Risk of bias analysis: Cochrane risk of bias assessment tool will be used to assess the risk of bias in the includedRCTs.Modified Jadad scale will be used to evaluate the quality of RCTs.

Strategy of data synthesis: Revman $\mathbf{5 . 3}$ software will be used to perform metaanalysis. The $Q$-test and 12 values will be applied to measure the inter-study heterogeneity. When the $\mathbf{P}$ value of $\mathbf{Q}$ test $>.1$ and $12<50 \%$, a fixed effects model will be applied; When $P$ value of $Q$ test $50 \%$, we will try to explore the causes of heterogeneity. If the heterogeneity is still significant, random effect model will be used for analysis. Binary variables were expressed using the odds ratio with 95\% confidence interval $(\mathrm{Cl})$ and continuous variables by the standardized mean difference with $95 \% \mathrm{CI}$.

Subgroup analysis: Subgroup analysis will be used to explore potential factors causing significant heterogeneity, such as intervention measures, control measures, study quality, sample size, course of treatment, risk of bias,etc.

Sensitivity analysis: We will try to eliminate the low-quality RCT according to the assessments results of risk of bias and methodological quality to confirm the stability of conclusion. If the overall quality of RCTs contained under the outcome are 
similar, the method of excluding RCT one by one will be used for sensitivity analysis.

Country(ies) involved: China.

Keywords: acupuncture, chronic obstructive pulmonary disease, metaanalysis, systematic review.

Contributions of each author:

Author 1 - Tianyi You.

Email: 568722548@qq.com

Author 2 - Haoyang Zhang.

Email: Inzyzhy@163.com

Author 3 - Jiquan Li.

Author 4 - Tieming Ma. 